\title{
Baljendra S. Kapoor, Jonathan M. Lorenz: Digestive disease interventions
}

\author{
Thieme, New York, Stuttgart, Delhi, Rio de Janeiro, 2018,307 p, format $22 \times 28.5 \mathrm{~cm}, 640$ \\ Illustrations, ISBN 978-1-62623-374-4, e ISBN 978-1-62623-375-1
}

\section{Bruno Grignon ${ }^{1}$}

Received: 1 August 2018 / Accepted: 4 August 2018 / Published online: 13 August 2018

(c) Springer-Verlag France SAS, part of Springer Nature 2018

Inspired by the enthusiasm and expertise of the meetings organized by the American Society of Digestive Disease Interventions and endorsed by the Society of Interventional Radiology, this work aimed at providing a comprehensive resource covering image-guided gastro-intestinal interventions of a full spectrum of pathologies.

It is composed of 30 chapters reviewing the multidisciplinary management of gastro-intestinal disorders with an emphasis on management options offered by interventional radiologists. A first chapter is devoted to recent advances in intraprocedural imaging, including in particular cone-beam computed tomography (CBCT), and navigational tools in practice. The second chapter deals with the role of multidetector computed tomography (MDCT) in two major vascular disorders, namely, acute gastro-intestinal hemorrhage and acute mesenteric ischemia, with a focus on appropriate scan protocols, imaging diagnosis, and diagnosis pitfalls. A more detailed chapter on mesenteric ischemia, including especially treatment options, is subsequently presented. Disease knowledge and management of portal hypertension and its complications are the topic of four succeeding chapters. The management of nonvariceal, respectively, upper and lower hemorrhages is presented in two specific chapters. The treatment of benign biliary strictures, malignant obstructive jaundice, acute cholecystis, cholelithiasis, and choledocholithiasis is the topic of three other chapters. An amount of 11 chapters is devoted to the state-of-the-art management of liver cancer and metastases, cholangiocarcinoma, and pancreatic cancer, including imaging, staging, ablation, tumor embolization, portal vein embolization, liver transplant complications, and Y-30. The interventional management of benign refractory ascites, percutaneous enteral access, colorectal obstruction, and intra-fluid collections is discussed in four specific chapters. Updates on recent advances and future directions such as bariatric embolization and islet cell transplantation are presented in two other chapters. A last chapter is devoted to pediatric gastro-intestinal interventions.

The authors are, respectively, Baljendra S. Kapoor, Associate Professor of Radiology, Cleveland Clinic Foundation, Cleveland, Ohio, USA, and Jonathan M. Lorenz, Professor of Radiology, Section of Interventional Radiology, University of Chicago, Chicago, Illinois, USA. A panel of 62 American contributors has collaborated in writing this work.

Up-to-date, richly illustrated, and clearly presented, this book will be of interest for all practitioners dealing with gastro-intestinal interventional radiology.

Author contributions BG: manuscript writing and editing.

\section{Compliance with ethical standards}

Conflict of interest The author declares that he has no conflict of interest.
Bruno Grignon

b.grignon@chu-nancy.fr

1 University of Lorraine, CHU Nancy, Nancy, France 